\title{
Characterization of Tissue-specific Transcriptomic Responses to Seedlessness Induction by Gibberellin in Table Grape
}

\author{
Soichiro Nishiyama ${ }^{1 * * * *}$, Daisuke Yoshimura ${ }^{2 * * *}$, Akihiko Sato ${ }^{3 * *}$ and Keizo Yonemori ${ }^{2}$ \\ ${ }^{1}$ Graduate School of Agriculture, Kyoto University, Kyoto 606-8502, Japan \\ ${ }^{2}$ Faculty of Agriculture, Ryukoku University, Otsu 520-2194, Japan \\ ${ }^{3}$ Grape and Persimmon Research Station, Institute of Fruit Tree and Tea Science, NARO, Higashihiroshima 739-2494, Japan
}

Gibberellins are widely used to induce seedlessness in table grape production, but are not effective for every cultivar or developmental stage. In order to characterize the potential physiological mechanism behind gibberellin-induced seedlessness, we conducted tissue-specific transcriptome analysis of ovules and ovaries from cultivars with different levels of receptiveness to seedlessness induction. $\mathrm{GA}_{3}$ treatment at full bloom highly induced gibberellin signaling and various phytohormone signaling pathway genes in ovules at two days after treatment, but had less effect in ovaries, which suggests that $\mathbf{G A}_{3}$ directly affects seed development. During the development to six days after $\mathbf{G A}_{3}$ application, cell cycle-related genes in the ovules were markedly downregulated, indicating that this may be related to ovule abortion induction. The marked transcriptomic responses were largely absent in a cultivar with low receptiveness to gibberellin-induced seedlessness, suggesting a crucial role for the observed tissue-specific responses in the successful induction of seedlessness by $\mathbf{G A}_{3}$ application. Collectively, our transcriptome analysis highlights distinct tissue-specific reactions to $\mathbf{G A}_{3}$ during early berry development, providing important insights for the successful production of seedless berries by gibberellin application.

Key Words: gene expression, phytohormone, RNA-seq, seed development.

\section{Introduction}

Phytohormones are signaling molecules in plants that control various physiological processes. Phytohormones are generally effective at low concentrations and have been intensively applied in agricultural practices (Jiang and Asami, 2018; Šimura et al., 2018). One of the most successful examples of the practical application of phytohormone is gibberellins (GAs) for seedless table grape (Vitis vinifera, V.labrusca, and their hybrids) production. GA application on developing flowers induces seed abortion and results in seedless berries (Ledbetter and Ramming, 1989). Also, GA application is routinely used to increase berry size and cluster thinning in the production of genetically

Received; August 16, 2021. Accepted; November 29, 2021.

First Published Online in J-STAGE on March 2, 2022.

Special Issue 'Reproductive biology of fruit and nut tree crops'.

* Corresponding author (E-mail: nishiyama.soichiro.8e@kyoto-u. ac.jp).

** Present address: Experimental Farm, Kindai University, Yuasa 643-0004, Japan.

*** These two authors contributed equally to this work. seedless (stenospermocarpic) cultivars (Ledbetter and Ramming, 1989; Acheampong et al., 2017). Currently, in Japan, seedlessness is a commercial standard for table grapes (Yamada and Sato, 2016). In many commercial cultivars, seedlessness is practically achieved by the application of $\mathrm{GA}_{3}$ at full bloom to three days after full bloom. For example, $\mathrm{GA}_{3}$ application at flowering is known to be effective to induce seedlessness for the tetraploid hybrids 'Kyoho' and 'Pione', and the diploid hybrid 'Shine Muscat' (Kishi and Tazaki, 1960; Fukunaga and Kurooka, 1988; Kondo and Kawai, 1998; Yamada et al., 2008).

GA-induced seedlessness for table grape was achieved firstly for 'Delaware' (V. labruscana) in the 1960s. Seedlessness in 'Delaware' is known to be achieved by applying $\mathrm{GA}_{3}$ at 14 days before anthesis, but if $\mathrm{GA}_{3}$ is applied around the flowering stage, seedlessness is not induced perfectly and the cluster consists of many seeded berries. Some studies suggested that the seedlessness of the 'Delaware' grape is attributed to the production of sterile pollen and delayed embryo development caused by $\mathrm{GA}_{3}$ application at 14 days before anthesis (Sugiura and Inaba, 1966; Sugiura, 1969). As 
mentioned above, it is also known that inducing seedlessness by applying $\mathrm{GA}_{3}$ for the cultivars bearing large-sized berries, such as 'Kyoho' and 'Pione', should be at full bloom to three days after full bloom, whereas it should be at 14 days before anthesis for small-sized berry cultivars such as 'Delaware'. Details on the seedlessness induction mechanisms for the large-sized berry remain unclear.

We previously showed that some cultivars with largesized berries can be divided into two types according to the process in GA-induced seedlessness (Yoshimura et al., 2021); one type is naturally difficult to fertilize due to abnormality of embryo development, and the other type can develop normal embryo. The former normally causes a large amount of berry drop around anthesis, but $\mathrm{GA}_{3}$ application from full bloom to three days after full bloom promotes fruit set and development, and produces seedless berries. 'Kyoho' and 'Pione' belong to this type. The latter type fertilizes and produces seeded berries in the cluster at a quite high rate in natural conditions; induced seedlessness by $\mathrm{GA}_{3}$ application as in the former type is also possible. 'Shine Muscat' and 'Honey Venus' belong to this type, but have different sensitivities to GA-induced seedlessness. Yoshimura et al. (2021) reported that a higher rate of seedless berries could be obtained in 'Shine Muscat' than in 'Honey Venus' in a $\mathrm{GA}_{3}$ treatment experiment. This difference in response to GA was suggested to be a heritable trait (Nagata and Kurihara, 1982). Due to the variation in responsiveness to GA, not all genetic resources are currently available for seedless berry production. Understanding the mechanisms of the GA response may have significant implications for largesized berry grape production and breeding.

GA is a phytohormone that is essential for many developmental processes in plants, including seed germination, stem elongation, leaf expansion, pollen maturation, and the induction of flowering (Achard and Genschik, 2009; Davière and Achard, 2013). GA signal transduction in Arabidopsis seeds positively regulates seed germination (Vishal and Kumar, 2018). In Arabidopsis, the molecular regulatory mechanisms of GA signaling for ovule initiation, integument development, and late embryogenesis control were uncovered (Gomez et al., 2016, 2018; Hu et al., 2018); these mechanisms may also be associated with the success of seedlessness induction by GA treatment in grapevines, but have not been examined in detail. GA signaling is often regulated by the amount of GA, and the levels of bioactive GAs in plants are maintained via feedback regulation of GA metabolism (Yamaguchi, 2008). GA2ox, which catabolizes active GAs, is often one of the major regulators of GA levels and is highly responsive to changes in GA signaling to ensure GA homeostasis (Rieu et al., 2008; Galinha et al., 2009; Sun, 2011). In contrast, expression of the genes encoding the GA biosynthesis enzymes GA20ox and GA3ox is high- ly elevated in a GA-deficient background, whereas these genes are downregulated after application of bioactive GAs in Arabidopsis (Phillips et al., 1995; Matsushita et al., 2007; Yamaguchi, 2008). It was suggested that this feedback regulation is conserved in grapevines, at least in developing berries ( $\mathrm{He}$ et al., 2019).

Furthermore, previous reports suggested the potential molecular mechanisms underlying seedlessness induction by pre-bloom GA application. Cheng et al. (2015) reported changes in the expression of genes for the biosynthesis and signaling of phytohormones, including GA, jasmonic acid, and salicylic acid, various transcription factors, and secondary metabolites by $\mathrm{GA}_{3}$ treatment at 12 days before flowering in 'Kyoho'. In addition, Cheng et al. (2013) reported changes in redox homeostasis by $\mathrm{GA}_{3}$ treatment at 6-12 days after treatment in 'Kyoho', 'Red Globe', and 'Thompson Seedless' berries treated 16-18 days before flowering. The above studies showed general features of physiological responses upon $\mathrm{GA}_{3}$ application; however, the following points are still not clear: (1) the key process responsible for GA-induced seedlessness, (2) the tissuespecificity of the responses caused by GA, and (3) the mechanisms underlying the differential response among large-sized berry grape cultivars, e.g., between 'Shine Muscat' and 'Honey Venus'.

In this study, the details of the response to $\mathrm{GA}_{3}$ treatment were analyzed with tissue-specific transcriptome analysis of large-sized berry grape cultivars, 'Shine Muscat' and 'Honey Venus', that shows different levels of receptiveness to seedlessness induction. In particular, tissue-specific transcriptome profiles and GA signalingrelated genes were analyzed to discuss the variation in the $\mathrm{GA}_{3}$ response during the flowering period.

\section{Materials and Methods}

\section{Plant materials and GA treatment}

This study was conducted in the 2019 harvest season. Four-year-old 'Shine Muscat' (SM) plants, grafted on 'Kober 5 BB' grown at the Maki experimental farm of Ryukoku University (Otsu, Japan), were used in this study. Five SM plants grown in 30 -L pots were trained and cultivated using the short cane pruning cultivation system. The plants were cultivated with a conventional cultivation method (a similar method was presented in Suehiro et al., 2019). A few days before flowering, all flower buds in a cluster except for those bearing up to $3.5-4 \mathrm{~cm}$ from the tip were thinned, leaving approximately 45 flowers in each cluster. The cluster was dipped in a $25 \mathrm{ppm} \mathrm{GA}_{3}$ solution, which is a standard concentration for the production of cultivars bearing large-sized berry in Japan, on the day all the flowers in the cluster had opened. The no-GA 3 treatment clusters, which had the same bloom day as the treated clusters, were used as control.

We also analyzed six-year-old 'Honey Venus' (HV) 
and SM plants grafted on 'Kober 5 BB' in the orchard at the Grape and Persimmon Research Division, NARO Institute of Fruit Tree and Tea Science (HigashiHiroshima, Japan). One vine was used for each fieldgrown cultivar and trained using the long cane pruning cultivation system. $\mathrm{GA}_{3}$ application was conducted as mentioned above. Pedigrees of SM and HV are available in references (Yamada et al., 2003, 2008).

To investigate seedlessness in the $\mathrm{GA}_{3}$-treated and control clusters, clusters were collected in the harvest season (4th September) for the plants at Otsu, and just after véraison (3rd July) for the plants at HigashiHiroshima. The berries were cut with a razor blade to visually determine the presence or absence of seeds. The number of seeds per berry was counted and the percentage of seeded berries per cluster was calculated for each cluster.

For the analysis of transcriptome, ovule and ovary tissues were separately collected at 2 and 6 days after treatment (DAT) from the berries of SM (Otsu) and HV (Higashi-Hiroshima) (Supplementary Fig. S1). The samples were immediately frozen in liquid nitrogen and stored at $-80^{\circ} \mathrm{C}$ until use. We selected the 2 and 6 DAT stages because there was no obvious difference in ovule size between treatment and control at 2 DAT, whereas the difference began to be visible at 6 DAT. Five berries were used as one sample, and four or five biological replicates were provided for RNA-Seq analysis. It should be noted that at least six days after flowering, there was no visible difference in the size and morphology of ovules and ovary tissues between the two cultivars in the control samples.

\section{RNA extraction, library preparation, and sequencing}

Total RNA was isolated by the hot borate method (Wan and Wilkins, 1994) from the ovule and ovary samples and quantified using Qubit 4 Fluorometer (Invitrogen, Carlsbad, CA, USA). Five hundred nanograms of total RNA were used to prepare mRNAseq libraries. Double-stranded cDNA was prepared and fragmented according to a previous report (BurkartWaco et al., 2013). The fragmented cDNA was subsequently processed with the GenNext ${ }^{\circledR}$ NGS Library Prep Kit (TOYOBO, Osaka, Japan) and the TruSeq DNA CD Indexes (Illumina, San Diego, CA, USA) for library preparation. Seventy-nine libraries in total were multiplexed and sequenced with three lanes of the Illumina HiseqX system (PE150) at Macrogen (Daejeon, Korea). All RNA samples used for the RNAseq analysis are listed in Supplementary Table S1. All sequence data are available in the DDBJ Sequenced Read Archive (accession number DRA011277).

\section{RNA-seq data analysis}

All sequences were pre-processed using fastp (Chen et al., 2018) with a minimum read length threshold of $35 \mathrm{bp}$. The reads from each library were aligned to the coding sequence of the $12 \mathrm{X}$ Vitis vinifera reference genome with version 2.1 gene annotation (Jaillon et al., 2007), which was available at Phytozome v13<https:// phytozome-next.jgi.doe.gov/info/Vvinifera_v2_1>, using BWA-MEM with default parameters ( $\overline{\mathrm{Li}}$ and Durbin, 2009). Low-quality alignments were filtered out by using SAMtools view with -q 20 option ( $\mathrm{Li}$ et al., 2009). All libraries were checked to ensure they had mapped read counts greater than one million. The number of reads mapped to each contig was counted from the SAM files with a custom python script: when one read was mapped to multiple genes, it was counted for each gene, i.e., there could be multiple counts for one read (Nishiyama et al., 2018). Gene expression level was represented as reads per kilobase per million reads (RPKM).

The DESeq2 package (Love et al., 2014) was used to define differentially expressed genes (DEGs) between GA-treated and control samples with a threshold of FDR $<0.05$. The DEGs were hierarchically clustered by Morpheus software <https://software.broadinstitute. org/morpheus $>$ with the average-linkage method, using a matrix of mean RPKM values of the biological replicates.

In addition to the functional annotations from the v2.1 database, the amino acid sequences of the annotated genes were searched against the representative protein sequences of the Araport11 Arabidopsis database with blastp. The best hit with an e-value threshold $<\mathrm{e}^{-20}$ was retrieved for each gene. A unique list of the GO terms from the v2.1 annotations and the GO terms assigned to the best-hit Arabidopsis locus was prepared and used for GO enrichment analysis. GO enrichment analysis was conducted using the topGO (version 2.24.0; <https://bioconductor.org/packages/release/bioc/ html/topGO.html $>$ ) R package with the weight01 algorithm and the option for Fisher's exact test. GO terms with $P$-values $<0.05$ and with gene counts $>2$ were considered enriched GO terms.

\section{Results}

\section{Effect of GA application on seed development}

The rates of seeded berries per cluster (Fig. 1A), as well as the number of seeds per berry (Fig. 1B), were significantly lower in the $\mathrm{GA}_{3}$ treatments than the controls in both SM (from Otsu and Higashi-Hiroshima) and $\mathrm{HV}$ (from Higashi-Hiroshima). Moreover, $\mathrm{GA}_{3}$ treatment induced more seedless berries in SM than in HV.

\section{Tissue-specific transcriptome profile}

Principal component analysis showed that biological replicates were plotted close together without exception (Supplementary Fig. S2). Along with the first principal component, samples were separated at 2 and 6 DAT in both ovules and ovaries of SM, whereas no cluster separation was associated with $\mathrm{GA}_{3}$ treatment. Although 
SM (loc1, Otsu) and HV (Higashi-Hiroshima) had different genetic backgrounds, sampling locations, and management, the plots of the principal component analysis almost overlapped and there was no clear separation of cultivars.

In the differential expression analysis, 3,739 and 1,798 unique DEGs were identified in SM and $\mathrm{HV}$, respectively, between the $\mathrm{GA}_{3}$-treated and control samples (Table 1). The number of DEGs increased in the samples at 6 DAT compared to the samples at 2 DAT, except for the HV ovary samples. Several DEGs overlapped between SM and HV ovules at 6 DAT, whereas the other samples showed very limited shared DEGs. In the following, the results obtained from SM, with highlevel receptiveness to $\mathrm{GA}_{3}$, are mainly described.

\section{Exploration of key systems involved in GA-induced seedlessness}

The 3,739 DEGs that responded to $\mathrm{GA}_{3}$ treatment in SM were divided into four clusters by hierarchical clustering (Fig. 2). Cluster 1 included genes that were highly expressed in control ovule samples. This cluster contained genes with basic functions such as "translation" (Supplementary Table S2). Cluster 2 showed a significant expression pattern in the ovule samples at 2 DAT and appeared to be an important cluster of genes associated with the very early response to $\mathrm{GA}_{3}$ treatment and the difference in $\mathrm{GA}_{3}$ responsiveness between $\mathrm{SM}$ and HV. The genes assigned to cluster 2 were enriched with functions related to various plant hormones, including ethylene, ABA, cytokinin, and salicylic acid, in addition to GA metabolism and signaling (Supplementary Table S3). Also, the expression of genes annotated with GO terms related to GA, found in cluster 2, was remarkably affected by $\mathrm{GA}_{3}$ treatment in the SM ovule sample, whereas most of the genes were not affected in the SM ovary (Fig. 3). Cluster 3 contained genes that were markedly differentially expressed in ovule samples at 6 DAT. Cluster 3 was characterized by a high number of genes involved in cell division and development (Supplementary Table S4) and may be associated with the key processes for abortion in $\mathrm{GA}_{3}$ response. Cluster 4 contained genes that were predominantly expressed in the ovary samples. This cluster contained genes involved in cell differentiation and stress responses (Supplementary Table S5).

We also characterized gene expression related to GA metabolic and signal transduction pathways. The expression of GA2ox, encoding an enzyme that catabolizes active GA, and of GA20ox and GA3ox, major GA biosynthesis genes, were investigated (Fig. 4). The expression levels of GA2ox and GA3ox were considerably higher than GA20ox. Particularly, markedly high expression of GA2ox was observed in the SM ovules and approximately doubled on average by $\mathrm{GA}_{3}$ treatment at 2 and 6 DAT. Variations in the expression of GA2ox in the SM control samples were large, and the
A

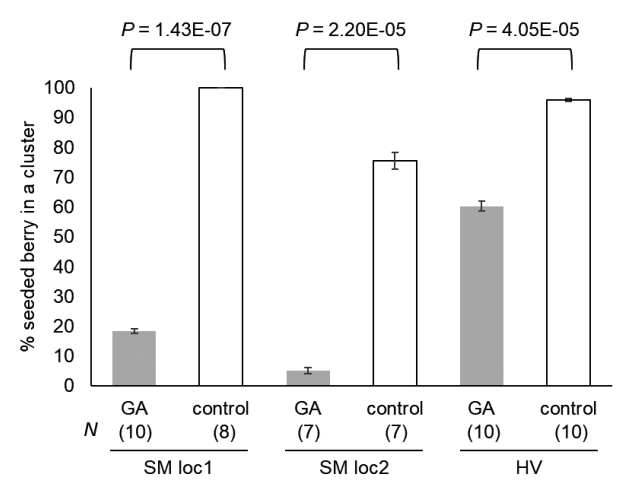

B

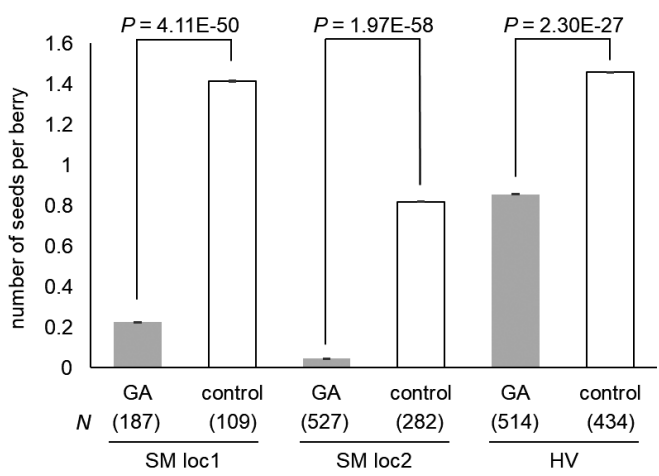

Fig. 1. Effect of $\mathrm{GA}_{3}$ treatment on seed development of 'Shine Muscat' (SM) and 'Honey Venus' (HV). GA 3 treatment was performed at flowering. A: Ratio of seeded berries per cluster. Student's $t$-test was conducted on the arcsine transformed value. B: Number of seeds per berry. loc1 represents samples harvested at Otsu on 4th Sep. loc2 and HV samples were harvested at Higashi-Hiroshima on 3rd Jul. Error bars indicate standard error. The numbers in parentheses indicate the number of clusters (A) and berries (B) tested.

Table 1. The number of differentially expressed genes (DEGs) between $\mathrm{GA}_{3}$-treated and control samples.

\begin{tabular}{|c|c|c|c|c|c|c|c|}
\hline \multirow[b]{2}{*}{ Sample } & \multicolumn{3}{|c|}{ Ovary } & \multicolumn{3}{|c|}{ Ovule } & \multirow{2}{*}{$\begin{array}{l}\text { Unique } \\
\text { counts of } \\
\text { total DEGs }\end{array}$} \\
\hline & $2 d$ & $6 \mathrm{~d}$ & $\begin{array}{c}\text { Shared between } \\
2 \mathrm{~d} \text { and } 6 \mathrm{~d}\end{array}$ & $2 \mathrm{~d}$ & $6 \mathrm{~d}$ & $\begin{array}{c}\text { Shared between } \\
2 \mathrm{~d} \text { and } 6 \mathrm{~d}\end{array}$ & \\
\hline Shine Muscat (SM) & 651 & 1087 & 68 & 664 & 2057 & 64 & 3739 \\
\hline Honey Venus (HV) & 328 & 181 & 15 & 355 & 1218 & 96 & 1798 \\
\hline Shared between SM and HV & 19 & 47 & 0 & 13 & 351 & 1 & 703 \\
\hline
\end{tabular}




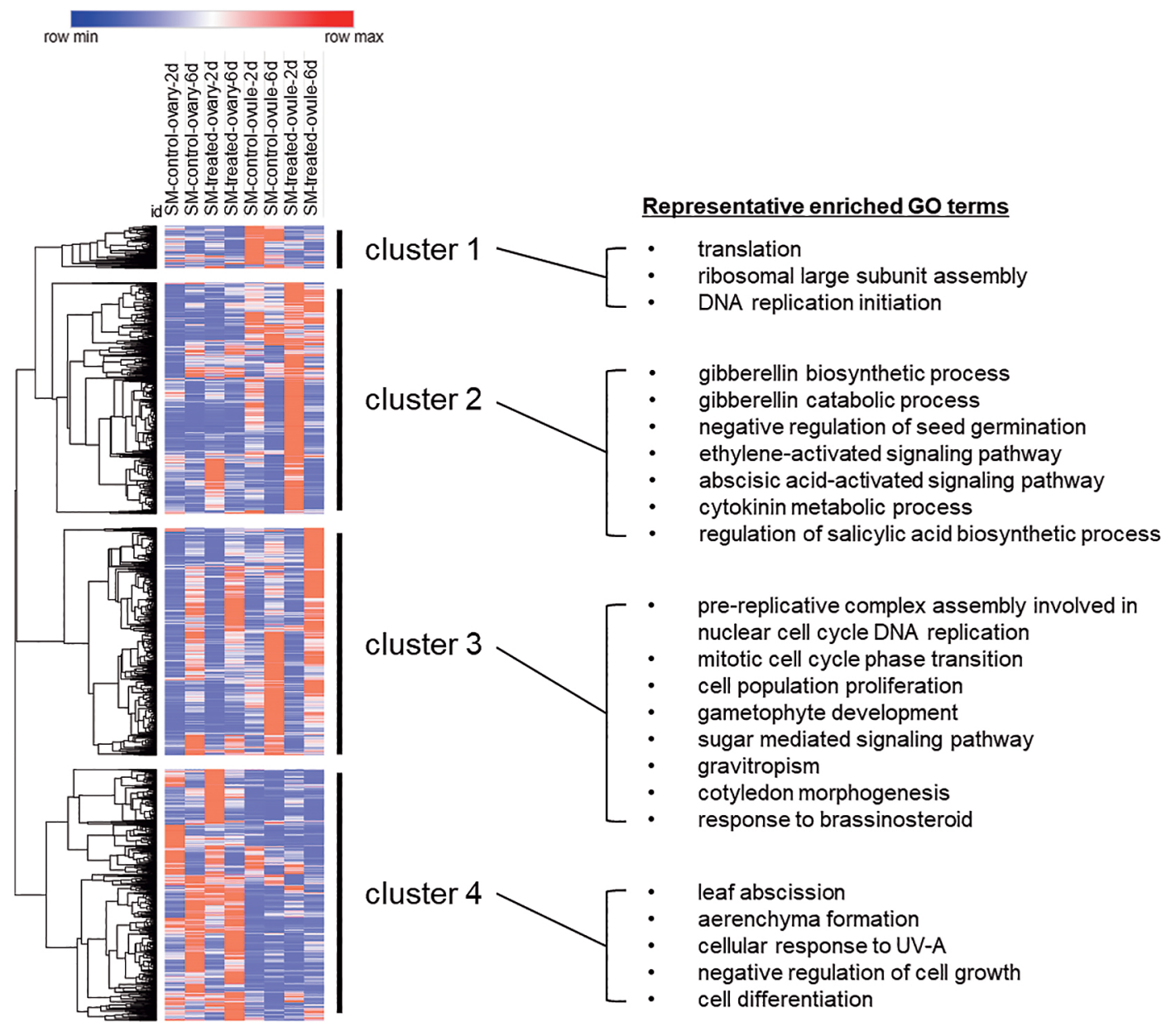

Fig. 2. Overview of expression patterns of the differentially expressed genes between the $\mathrm{GA}_{3}$-treated and control groups of the SM samples. Gene expression levels were represented as a heatmap based on the average RPKM value of each group, and the color scheme was normalized for each gene. Blue indicates low expression and red indicates high expression. Full lists of the enriched GO terms are shown in Supplementary Tables S2-S5.

effect of $\mathrm{GA}_{3}$ was statistically not significant. Notably, this response was not observed in the ovary tissue. In addition, expression of the GA biosynthesis genes, GA20ox and GA3ox, was significantly changed by $\mathrm{GA}_{3}$ treatment in the SM ovary, but not in the SM ovules. Collectively, the GA catabolism gene was generally upregulated by $\mathrm{GA}_{3}$ application in the $\mathrm{SM}$ ovule, but the GA biosynthesis genes were not affected. In the ovary tissue, the presumed GA feedback system was less active than in the ovule tissues.

To identify the potential key genes responsible for seedlessness, the DEGs detected in the SM ovule samples were ranked by fold change and the top genes are shown in Table 2 . The highly upregulated genes included several ABA-related genes, such as MYB15, ABR1, $C B F 4$, and $H V A 22 A$, as well as genes related to somatic embryogenesis, such as EP3 and PSK4 (Table 2). Conversely, OFP16, a member of the OVATE family, and $S P X 2$, which is involved in phosphate signaling, were strongly downregulated by $\mathrm{GA}_{3}$ treatment (Table 3 ).

\section{Comparison of GA responses between HV and SM}

To validate the results obtained above, we referred to the $\mathrm{GA}_{3}$ response in the low-level receptiveness cultivar HV. The feedback reaction of GA metabolism, which was typical in the SM ovules, was also observed in the HV ovules and ovary samples, but the early strong reaction of GA2ox at 2 DAT in the SM ovules was completely absent in the HV ovules (Fig. 4). In the HV samples, unlike SM, expression induction of GArelated genes by $\mathrm{GA}_{3}$ treatment was largely absent in the ovules and ovaries, except for GA2ox at 6 DAT (Fig. 4). The cell cycle-related DEGs detected in the SM ovules, which are thought to be important for abortion in response to $\mathrm{GA}_{3}$ (Fig. 2), were not differentially expressed in the HV ovules (Fig. 5).

\section{Discussion}

Tissue-specific transcriptome reactions to GA in grape flowers

The effect of $\mathrm{GA}_{3}$ application on seed development was clear and highly significant. $\mathrm{GA}_{3}$ application was effective in inducing seedlessness in both SM and HV, 


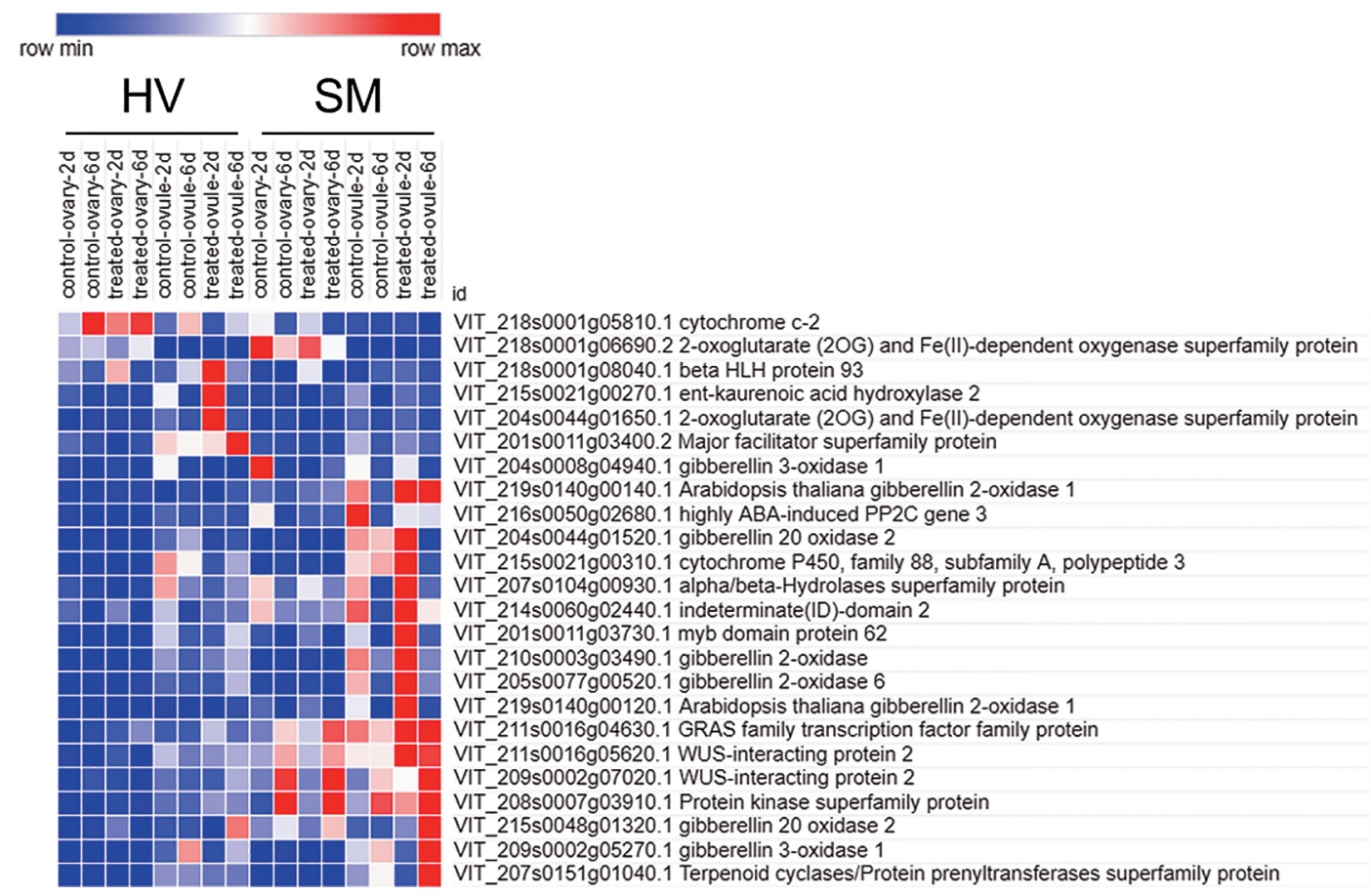

Fig. 3. Expression profiles of GA-related DEGs. DEGs with the GO terms "gibberellin biosynthetic process" (GO:0009686), "gibberellin catabolic process" (GO:0045487), "regulation of gibberellic acid mediated signaling pathway" (GO:0009937), and "gibberellic acid homeostasis" (GO:0010336) are shown. Gene expression levels were represented as a heatmap based on the average RPKM value of each group, and the color scheme was normalized for each gene. Blue indicates low expression and red indicates high expression. The GO terms annotated for each gene are listed in Supplementary Table S6.

although SM was more receptive and was almost entirely seedless when treated with $\mathrm{GA}_{3}$ (Fig. 1). This result was consistent with previous reports (Yamada et al., 2008; Satoyoshi et al., 2015; Yoshimura et al., 2021). GA-induced seedlessness by the same method as in this study has been widely applied in conventional cultivation of table grapes in Japan (Yamada and Sato, 2016), and it is empirically known that despite some cases where seeds are accidentally developed in some conditions, even with $\mathrm{GA}_{3}$ application in the $\mathrm{GA}_{3}$-receptive cultivars, the effect of GA-induced seedlessness is highly stable across years, even in with a different environment and juvenility. We observed this robustness in the SM experiment conducted at two locations (Fig. 1). Therefore, although we collected RNA from berry samples from two locations (SM from Otsu, and HV from Higashi-Hiroshima), we could make a fair comparison on the $\mathrm{GA}_{3}$ response between the two samples. It should be noted that since Vitis generally has two ovules per carpel and two carpels in a flower, there could be four seeds in a berry; whereas it was observed that even in the control a berry contained only approximately 1.4 seeds on average (Fig. 1). Houel et al. (2013) reported that the average seed number per berry over hundreds of cultivars was 1.9 , with great variation by cultivar. The lower seed formation rate in natural conditions suggests that GA-induced seedlessness is a mixture of the natural abortion process and GA responsive process. The latter was focused on in this study and was assumed to be measurable by comparing cultivars with the same level of seed formation ability, which was observed in the control samples (Fig. 1).

The result showed that the reaction to $\mathrm{GA}_{3}$ treatment is distinct and most likely independent between ovaries and ovules. In the SM samples with high sensitivity to $\mathrm{GA}_{3}$, dramatic expression changes in phytohormonerelated genes were observed in the ovules at 2 DAT (Figs. 2 and 3). The strong response to $\mathrm{GA}_{3}$ at this early stage is consistent with previous studies investigating the whole flower transcriptome of genetically seeded cultivars (Cheng et al., 2015). Conversely, this response was largely absent in the ovaries (Figs. 2 and 3). A similar pattern was also observed in the GA biosynthesis and catabolism genes (Fig. 4). In the SM ovule samples, GA2ox, which encodes an enzyme that catabolizes active GAs, was highly expressed and was upregulated at 2 DAT in response to $\mathrm{GA}_{3}$ application. Collectively, the expression of the GA metabolism genes was regulated towards balancing the GA level in tissues. The response of the GA2ox gene expression to $\mathrm{GA}_{3}$ was distinct in the SM ovule at 2 DAT, but it was not obvious in the SM ovaries. Taken together, these results showed that the responses to $\mathrm{GA}_{3}$ differed between ovary and ovule tissues, with the ovules being more responsive.

The present study showed that the dynamic functional changes in response to $\mathrm{GA}_{3}$ in early developing berries were mainly ovule (or seed trace) -specific reactions (Fig. 2; Supplementary Table S3), and this is a key 
A

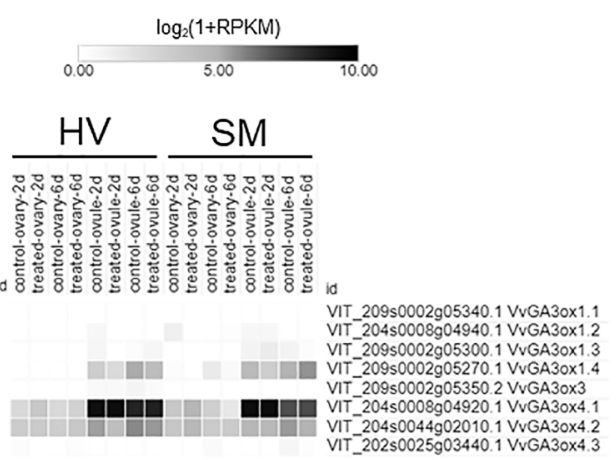

C

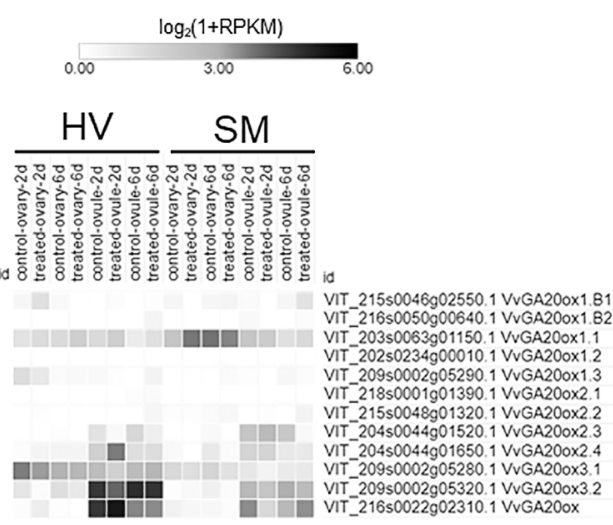

E

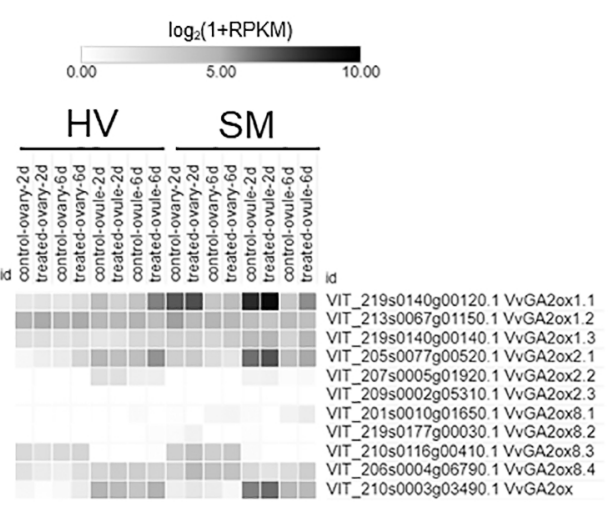

B

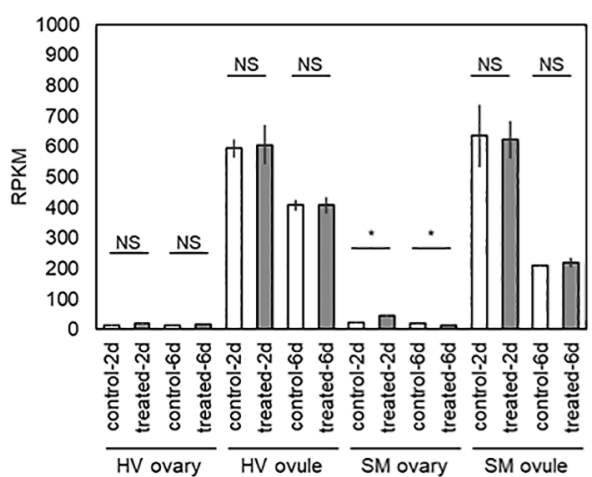

D

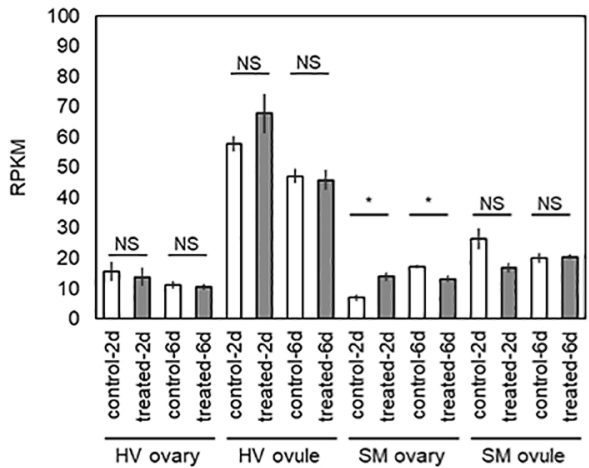

F

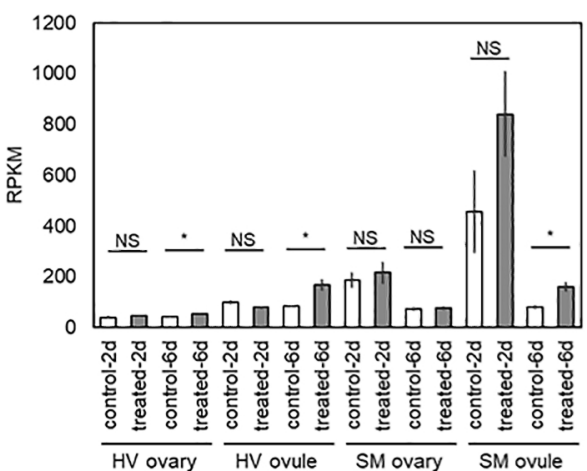

Fig. 4. Expression pattern of genes annotated as GA3ox (A, B), GA20ox (C, D), and GA2ox (E, F) in developing ovules and ovaries. The gene ID is based on the $12 \mathrm{X}$ Vitis vinifera reference genome with version 2.1 gene annotation (Jaillon et al., 2007), and the functional annotation of GA oxidases by Wang et al. (2020) was applied. A, C, E: $\log _{2}(1+$ RPKM) scores of each GA oxidase gene. B, D, F: Sum of RPKM values of GA oxidase paralogs. Error bars represented standard error. Asterisks indicated the statistical significance $(P<0.05)$ based on Student's $t$-test for sum of homologous genes.

finding for understanding seed and berry development. The importance of exogenous and endogenous GAs in early berry growth has been repeatedly highlighted in genetically seedless grape production (Cheng et al., 2013; Ravest et al., 2017). Differential expression of various phytohormone-related genes in response to $\mathrm{GA}_{3}$ application (Fig. 2; Supplementary Table S3) was also observed in studies on $\mathrm{GA}_{3}$ treatment of stenospermocarpic cultivars based on a whole-berry analysis, and was previously considered a cause of berry enlargement induced by $\mathrm{GA}_{3}$ application (Chai et al., 2014;
Upadhyay et al., 2018). The functional components responsive to $\mathrm{GA}_{3}$ application reported by Chai et al. (2014), Upadhyay et al. (2018), and Cheng et al. (2015), based on whole-berry analysis fairly matched with those observed in the present study, suggesting that the responses observed in the previous studies, although at different stages, might track signals mainly from the ovule tissue. Collectively, for the above reasons, the hypothesis of the importance of the differential expression of phytohormone signaling genes in the GA-induced berry enlargement needs to be reformulat- 


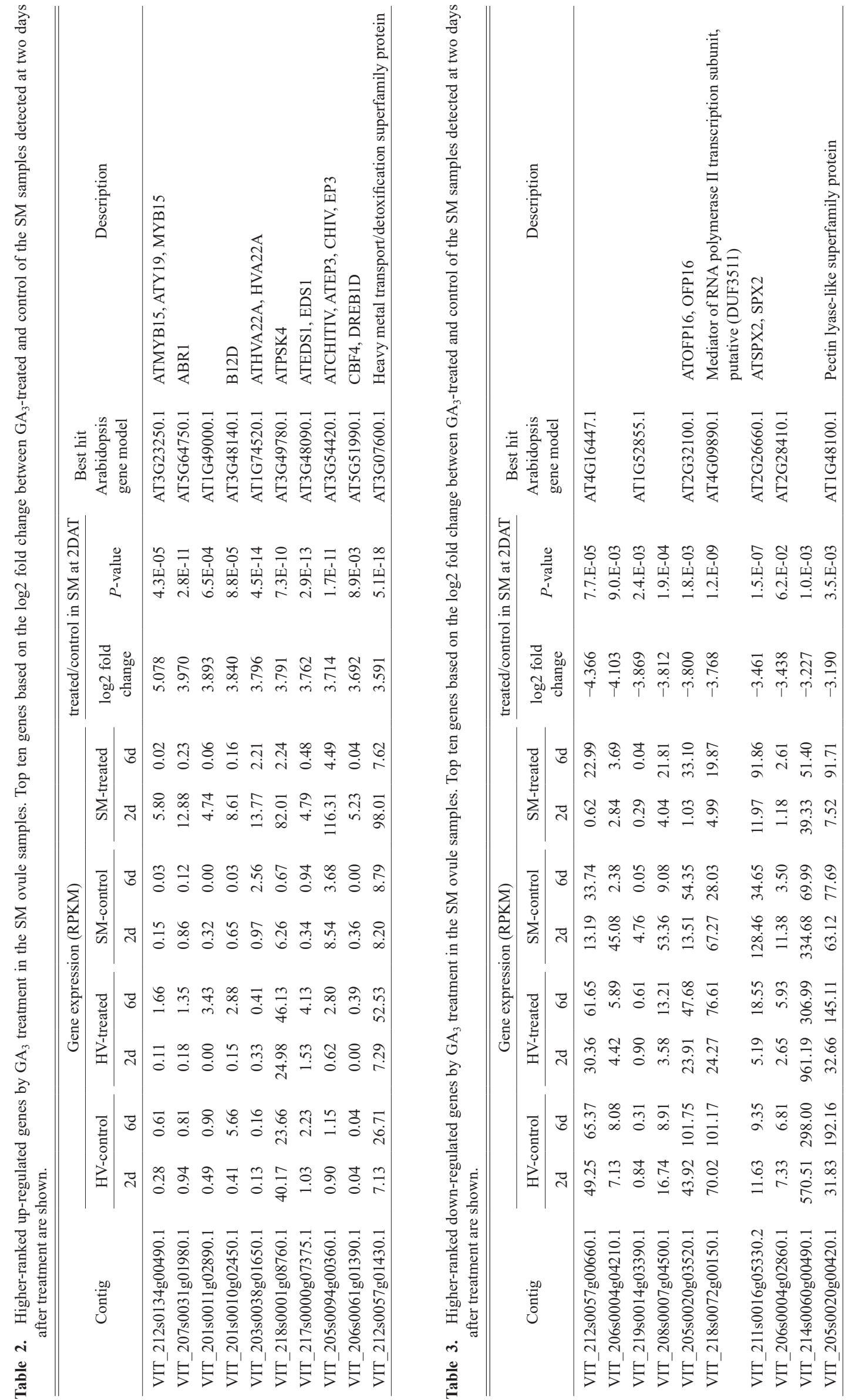




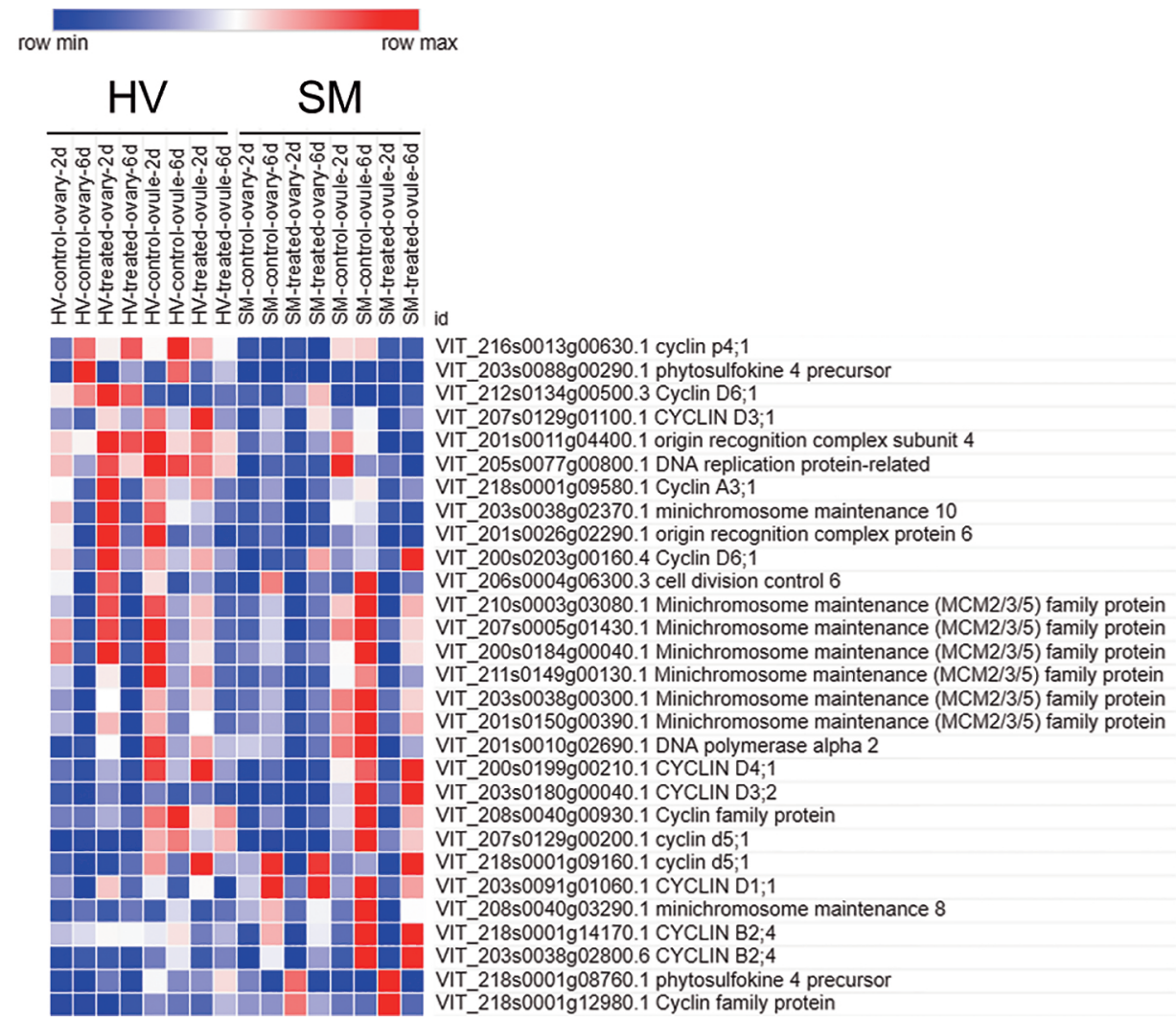

Fig. 5. Expression profile of the cell cycle-related DEGs detected in the comparisons of ovule samples for SM and HV. DEGs with the GO terms "cell population proliferation" (GO:0008283), "regulation of cyclin-dependent protein serine/threonine kinase activity" (GO:0000079), and "DNA replication initiation" (GO:0006270) are shown. Gene expression levels were represented as a heatmap based on the average RPKM value of each group, and the color scheme was normalized for each gene. Blue indicates low expression and red indicates high expression. The GO terms annotated for each gene were listed in Supplementary Table S7.

ed. Correa et al. (2015) reported a correlation between seed number and berry size in an $\mathrm{F}_{1}$ population, whereas this correlation was not significant under cultivation with $\mathrm{GA}_{3}$ application. Correa et al. (2015) interpreted that the correlation between seed number and berry size is caused by GA from mature seeds, and that exogenous $\mathrm{GA}_{3}$ application masked the GA variation in berry among the $\mathrm{F}_{1}$ individuals. This is partially supported by our results, demonstrating markedly lower GA metabolism in developing ovaries than ovules in early developing berries (Fig. 4). Unfortunately, we could not test the detailed effects of $\mathrm{GA}_{3}$ application on berry development in the present study; however, a similar sampling scheme as in the present study should result in a deeper understanding of berry size modulation by $\mathrm{GA}_{3}$ application, as well as seed development.

Various phytohormones and cell cycle control as possible key systems in GA-induced seedlessness

According to the results, a key process of the $\mathrm{GA}_{3}$ response in ovules was suggested to be the signaling of various phytohormones. In ovule tissue, the GA-related response occurred at 2 DAT, followed by a strong change in the expression of cell cycle-related genes at 6 DAT, suggesting that a key molecular response driving seed abortion functioned within 6 DAT (Figs. 3 and 5). Interestingly, the responses of various phytohormonerelated genes in the ovule tissue were observed at 2 DAT, including ethylene, ABA, cytokinin, and salicylic acid, as well as GA (Fig. 2; Supplementary Table S3). In particular, the salicylic acid-dependent defense mechanism was suggested to be involved in genetic seedlessness and may be the key process for seedlessness (Royo et al., 2018). As mentioned above, the response of phytohormones was reported for $\mathrm{GA}_{3}$ treated stenospermocarpic cultivars based on a wholeberry analysis (Chai et al., 2014; Upadhyay et al., 2018). Additionally, seed dry weight of stenospermocarpic individuals was reduced by $\mathrm{GA}_{3}$ application (Correa et al., 2015), suggesting that the same system observed in this study may function in diverse grapes, including genetically seedless plants. Collectively, the presented results and all the literature support the key role of the phytohormone signaling changes in GAinduced seedlessness.

Extensive expression changes in genes related to embryo development and seed dormancy or germination were also detected in $\mathrm{GA}_{3}$-treated ovule samples (Fig. 2; Tables 2 and 3). OFP16, a member of OVATE family proteins (OFPs), was markedly downregulated 
by $\mathrm{GA}_{3}$ treatment (Table 3). OFP is a regulator of various aspects of plant growth and development, and some OFP members are known to have a role in embryo development in Arabidopsis (Pagnussat et al., 2007; Wang et al., 2011, 2019). Furthermore, MYB15, CBF, and $A B R 1$, all of which are associated with ABA signaling, were strongly induced by $\mathrm{GA}_{3}$ treatment (Table 2). MYB15 and CBF function in cold acclimation, and MYB15 in Arabidopsis act as a transcriptional repressor of $C B F$, depending on the phosphorylation status of MYB15 (Agarwal et al., 2006; Kim et al., 2017). It is known that the $\mathrm{CBF}$ signaling pathway interacts with ABA signaling (Knight et al., 2004; Roychoudhury et al., 2013). Also, ABR1 in Arabidopsis is known to act as a transcriptional repressor of ABA response and has a role in seed germination (Pandey et al., 2005; Li et al., 2016). In addition, EP3 and PSK are known to affect somatic embryogenesis processes (De Jong et al., 1992; Igasaki et al., 2003). Taken together, these results suggest $\mathrm{GA}_{3}$ treatment may initially cause dynamic reprogramming of the developmental process in ovule tissue, followed by the alteration of cell division and subsequent seed abortion.

Potential mechanism of low sensitivity to GA-induced seedlessness in 'Honey Venus'

Many potential processes affect seedlessness by GA, from GA recognition to abortion, but the key process responsible for the variation in the GA response in seeds has never been identified. The process needs to be systemic enough to suppress the entire GA response reaction upon GA application, as observed in GA signal transduction (Fig. 3) and GA biosynthesis/catabolism (Fig. 4). The HV ovules largely lacked the dynamic $\mathrm{GA}_{3}$-responsive reactions observed in the $\mathrm{SM}$ ovules (Fig. 3), suggesting that this genetic variation may be conferred by a process before GA signal transduction in the ovules, rather than in downstream processes. Further work on the $\mathrm{GA}_{3}$ response with a wider range of variation may be beneficial for the breeding of cultivars suitable for seedlessness induction.

\section{Conclusion}

This study showed that $\mathrm{GA}_{3}$ application induces not only GA signaling, but also various phytohormone signaling genes in developing grape flowers. This response was predominantly observed in ovules, but not in ovaries; therefore, it may be inferred that the key system for seedlessness is ovule-specific. Cell cyclerelated genes in the ovules were markedly affected in the high gibberellin-sensitivity cultivar SM and may be an important process for ovule abortion. A marked response was observed in the processes of phytohormones and cell cycle genes, and these may be the initial targets for future genetic studies. Although this study showed valid results for only one $\mathrm{GA}_{3}$-responsive genotype, based on the literature, the results may be general- izable, and it is potentially meaningful to validate the results with a wider range of genotypes in the future. The results presented in this study provided a basic knowledge of the mechanism behind seedlessness induction, which is crucial for improving seedless table grape production.

\section{Literature Cited}

Achard, P. and P. Genschik. 2009. Releasing the brakes of plant growth: how GAs shutdown DELLA proteins. J. Exp. Bot. 60: 1085-1092.

Acheampong, A. K., C. Zheng, T. Halaly, L. Giacomelli, Y. Takebayashi, Y. Jikumaru, Y. Kamiya, A. Lichter and E. Or. 2017. Abnormal endogenous repression of GA signaling in a seedless table grape cultivar with high berry growth response to GA application. Front. Plant Sci. 8: 850. DOI: 10.3389/fpls.2017.00850.

Agarwal, M., Y. Hao, A. Kapoor, C. H. Dong, H. Fujii, X. Zheng and J. K. Zhu. 2006. A R2R3 type MYB transcription factor is involved in the cold regulation of CBF genes and in acquired freezing tolerance. J. Biol. Chem. 281: 3763637645.

Burkart-Waco, D., K. Ngo, B. Dilkes, C. Josefsson and L. Comai. 2013. Early disruption of maternal-zygotic interaction and activation of defense-like responses in Arabidopsis interspecific crosses. Plant Cell 25: 2037-2055.

Chai, L., Y. Li, S. Chen, A. Perl, F. Zhao and H. Ma. 2014. RNA sequencing reveals high resolution expression change of major plant hormone pathway genes after young seedless grape berries treated with gibberellin. Plant Sci. 229: 215224.

Chen, S., Y. Zhou, Y. Chen and J. Gu. 2018. fastp: an ultra-fast all-in-one FASTQ preprocessor. Bioinformatics 34: i884i890.

Cheng, C., C. Jiao, S. D. Singer, M. Gao, X. Xu, Y. Zhou, Z. Li, Z. Fei, Y. Wang and X. Wang. 2015. Gibberellin-induced changes in the transcriptome of grapevine (Vitis labrusca $\times$ V. vinifera) cv. Kyoho flowers. BMC Genomics 16: 1-16.

Cheng, C., X. Xu, S. D. Singer, J. Li, H. Zhang, M. Gao, L. Wang, J. Song and X. Wang. 2013. Effect of $\mathrm{GA}_{3}$ treatment on seed development and seed-related gene expression in grape. PLoS One 8: e80044. DOI: 10.1371/journal.pone. 0080044.

Correa, J., G. Ravest, D. Laborie, M. Mamani, E. Torres, C. Muñoz, M. Pinto and P. Hinrichsen. 2015. Quantitative trait loci for the response to gibberellic acid of berry size and seed mass in tablegrape (Vitis vinifera L.). Aust. J. Grape Wine Res. 21: 496-507.

Davière, J. M. and P. Achard. 2013. Gibberellin signaling in plants. Development 140: 1147-1151.

De Jong, A. J., J. Cordewener, F. Lo Schiavo, M. Terzi, J. Vandekerckhove, A. Van Kammen and S. C. De Vries. 1992. A carrot somatic embryo mutant is rescued by chitinase. Plant Cell 4: 425-433.

Fukunaga, S. and H. Kurooka. 1988. Studies on seedlessness of 'Kyoho' grapes induced by gibberellin in combination with streptomycin. Bull. Univ. Osaka Pref. Ser. B, Agric. Biol. 40: $1-10$.

Galinha, C., G. Bilsborough and M. Tsiantis. 2009. Hormonal input in plant meristems: A balancing act. Semin. Cell Dev. Biol. 20: 1149-1156.

Gomez, M. D., D. Barro-Trastoy, E. Escoms, M. Saura-Sánchez, I. Sánchez, A. Briones-Moreno, F. Vera-Sirera, E. Carrera, J. J. Ripoll, M. F. Yanofsky, I. Lopez-Diaz, J. M. Alonso and 
M. A. Perez-Amador. 2018. Gibberellins negatively modulate ovule number in plants. Development 145: dev163865. DOI: $10.1242 /$ dev.163865.

Gomez, M. D., D. Ventimilla, R. Sacristan and M. A. PerezAmador. 2016. Gibberellins regulate ovule integument development by interfering with the transcription factor ATS. Plant Physiol. 172: 2403-2415.

He, H., G. Liang, S. Lu, P. Wang, T. Liu, Z. Ma, C. Zuo, X. Sun, B. Chen and J. Mao. 2019. Genome-wide identification and expression analysis of GA2ox, GA3ox, and GA20ox are related to gibberellin oxidase genes in grape (Vitis Vinifera L.). Genes 10: 680. DOI: 10.3390/genes 10090680.

Houel, C., M. L. Martin-Magniette, S. D. Nicolas, T. Lacombe, L. Le Cunff, D. Franck, L. Torregrosa, G. Conéjéro, S. Lalet, P. This and A.-F. Adam-Blondon. 2013. Genetic variability of berry size in the grapevine (Vitis vinifera L.). Aust. J. Grape Wine Res. 19: 208-220.

Hu, Y., L. Zhou, M. Huang, X. He, Y. Yang, X. Liu, Y. Li and X. Hou. 2018. Gibberellins play an essential role in late embryogenesis of Arabidopsis. Nat Plants 4: 289-298.

Igasaki, T., N. Akashi, T. Ujino-Ihara, Y. Matsubayashi, Y. Sakagami and K. Shinohara. 2003. Phytosulfokine stimulates somatic embryogenesis in Cryptomeria japonica. Plant Cell Physiol. 44: 1412-1416.

Jaillon, O., J. M. Aury, B. Noel, A. Policriti, C. Clepet, A. Casagrande, N. Choisne, S. Aubourg, N. Vitulo, C. Jubin, A. Vezzi, F. Legeai, P. Hugueney, C. Dasilva, D. Horner, E. Mica, D. Jublot, J. Poulain, C. Bruyère, A. Billault, B. Segurens, M. Gouyvenoux, E. Ugarte, F. Cattonaro, V. Anthouard, V. Vico, C. Del Fabbro, M. Alaux, G. Di Gaspero, V. Dumas, N. Felice, S. Paillard, I. Juman, M. Moroldo, S. Scalabrin, A. Canaguier, I. Le Clainche, G. Malacrida, E. Durand, G. Pesole, V. Laucou, P. Chatelet, D. Merdinoglu, M. Delledonne, M. Pezzotti, A. Lecharny, C. Scarpelli, F. Artiguenave, M. E. Pè, G. Valle, M. Morgante, M. Caboche, A.-F. Adam-Blondon, J. Weissenbach, F. Quétier, P. Wincker and French-Italian Public Consortium for Grapevine Genome Characterization. 2007. The grapevine genome sequence suggests ancestral hexaploidization in major angiosperm phyla. Nature 449: 463-467.

Jiang, K. and T. Asami. 2018. Chemical regulators of plant hormones and their applications in basic research and agriculture. Biosci. Biotechnol. Biochem. 82: 1265-1300.

Kim, S. H., H. S. Kim, S. Bahk, J. An, Y. Yoo, J. Y. Kim and W. S. Chung. 2017. Phosphorylation of the transcriptional repressor MYB15 by mitogen-activated protein kinase 6 is required for freezing tolerance in Arabidopsis. Nucleic Acids Res. 45: 6613-6627.

Kishi, T. and M. Tazaki. 1960. Gibberellin application on Delaware grapes. Agric. Hort. 35: 381-384 (In Japanese).

Knight, H., D. G. Zarka, H. Okamoto, M. F. Thomashow and M. R. Knight. 2004. Abscisic acid induces CBF gene transcription and subsequent induction of cold-regulated genes via the CRT promoter element. Plant Physiol. 135: 1710 1717.

Kondo, S. and M. Kawai. 1998. Relationship between free and conjugated ABA levels in seeded and gibberellin-treated seedless, maturing 'Pione' grape berries. J. Amer. Soc. Hort. Sci. 123: 750-754.

Ledbetter, C. A. and D. W. Ramming. 1989. Seedlessness in Grapes. Hortic. Rev. 11: 159-184.

Li, H. and R. Durbin. 2009. Fast and accurate short read alignment with Burrows-Wheeler transform. Bioinformatics 25: $1754-1760$

Li, H., B. Handsaker, A. Wysoker, T. Fennell, J. Ruan, N. Homer,
G. Marth, G. Abecasis and R. Durbin. 2009. The Sequence Alignment/Map format and SAMtools. Bioinformatics 25: 2078-2079.

Li, T., X. Y. Wu, H. Li, J. H. Song and J. Y. Liu. 2016. A dualfunction transcription factor, AtYY1, is a novel negative regulator of the Arabidopsis ABA response network. Mol. Plant 9: 650-661.

Love, M. I., W. Huber and S. Anders. 2014. Moderated estimation of fold change and dispersion for RNA-seq data with DESeq2. Genome Biol. 15: 550. DOI: 10.1186/s13059-0140550-8.

Matsushita, A., T. Furumoto, S. Ishida and Y. Takahashi. 2007. AGF1, an AT-hook protein, is necessary for the negative feedback of AtGA3oxl encoding GA 3-oxidase. Plant Physiol. 143: 1152-1162.

Nagata, K. and A. Kurihara. 1982. The varietal difference in the response of grape cultivars to gibberellin application. Bull. Fruit Tree Res. Sta. Series E, Akitsu 4: 7-19 (In Japanese with English abstract).

Nishiyama, S., N. Onoue, A. Kono, A. Sato, K. Yonemori and R. Tao. 2018. Characterization of a gene regulatory network underlying astringency loss in persimmon fruit. Planta 247: 733-743.

Pagnussat, G. C., H. J. Yu and V. Sundaresan. 2007. Cell-fate switch of synergid to egg cell in Arabidopsis eostre mutant embryo sacs arises from misexpression of the BEL1-like homeodomain gene BLH1. Plant Cell 19: 3578-3592.

Pandey, G. K., J. J. Grant, Y. H. Cheong, B. G. Kim, L. Li and S. Luan. 2005. ABR1, an APETALA2-domain transcription factor that functions as a repressor of $\mathrm{ABA}$ response in Arabidopsis. Plant Physiol. 139: 1185-1193.

Phillips, A. L., D. A. Ward, S. Uknes, N. E. Appleford, T. Lange, A. K. Huttly, P. Gaskin, J. E. Graebe and P. Hedden. 1995. Isolation and expression of three gibberellin 20-oxidase cDNA clones from Arabidopsis. Plant Physiol. 108: 10491057.

Ravest, G., M. Mamani, L. Giacomelli, C. Moser, C. Pastenes and P. Hinrichsen. 2017. Bioactive gibberellins show differential abundance at key phenological stages for berry growth in table grapes. Am. J. Enol. Vitic. 68: 478-484.

Rieu, I., S. Eriksson, S. J. Powers, F. Gong, J. Griffiths, L. Woolley, R. Benlloch, O. Nilsson, S. G. Thomas, P. Hedden and A. L. Phillips. 2008. Genetic analysis reveals that C19GA 2-oxidation is a major gibberellin inactivation pathway in Arabidopsis. Plant Cell 20: 2420-2436.

Roychoudhury, A., S. Paul and S. Basu. 2013. Cross-talk between abscisic acid-dependent and abscisic acid-independent pathways during abiotic stress. Plant Cell Rep. 32: 985-1006.

Royo, C., R. Torres-Pérez, N. Mauri, N. Diestro, J. A. Cabezas, C. Marchal, T. Lacombe, J. Ibáñez, M. Tornel, J. Carreño, J. M. Martínez-Zapater and P. Carbonell-Bejerano. 2018. The major origin of seedless grapes is associated with a missense mutation in the MADS-box gene VviAGL11. Plant Physiol. 177: 1234-1253.

Satoyoshi, Y., Y. Udo, N. Saito and M. Mitsumori. 2015. High quality grape production with newly released cultivars. Bull. Yamanashi Fruit Tree Exp. Sta. 14: 55-65 (In Japanese).

Šimura, J., I. Antoniadi, J. Široká, D. Tarkowská, M. Strnad, K. Ljung and O. Novák. 2018. Plant hormonomics: multiple phytohormone profiling by targeted metabolomics. Plant Physiol. 177: 476-489.

Suehiro, Y., K. Mochida, M. Tsuma, Y. Yasuda, H. Itamura and T. Esumi. 2019. Effects of gibberellic acid/cytokinin treatments on berry development and maturation in the yellow-green skinned 'Shine Muscat' grape. Hort. J. 88: 202-213. 
Sugiura, A. 1969. Gibberellin-induced seedlessness of grapes. Chem. Reg. Plant. 4: 63-67 (In Japanese).

Sugiura, A. and A. Inaba. 1966. Studies on the mechanism of gibberellin-induced seedlessness of Delaware grapes. I. J. Japan. Soc. Hort. Sci. 35: 233-241.

Sun, T. P. 2011. The molecular mechanism and evolution of the GA-GID1-DELLA signaling module in plants. Curr. Biol. 21: R338-R345.

Upadhyay, A., S. Maske, S. Jogaiah, N. Y. Kadoo and V. S. Gupta. 2018. $\mathrm{GA}_{3}$ application in grapes (Vitis vinifera L.) modulates different sets of genes at cluster emergence, full bloom, and berry stage as revealed by RNA sequence-based transcriptome analysis. Funct. Integr. Genomics 18: 439455.

Vishal, B. and P. P. Kumar. 2018. Regulation of seed germination and abiotic stresses by gibberellins and abscisic acid. Front. Plant Sci. 9: 838. DOI: 10.3389/fpls.2018.00838.

Wan, C. Y. and T. A. Wilkins. 1994. A modified hot borate method significantly enhances the yield of high-quality RNA from cotton (Gossypium hirsutum L.). Anal. Biochem. 223: $7-12$.

Wang, S., Y. Chang, J. Guo, Q. Zeng, B. E. Ellis and J. G. Chen. 2011. Arabidopsis ovate family proteins, a novel transcriptional repressor family, control multiple aspects of plant growth and development. PLoS One 6: e23896. DOI: 10.1371/journal.pone.0023896.

Wang, W., Y. Bai, P. Koilkonda, L. Guan, Y. Zhuge, X. Wang, Z. Liu, H. Jia, C. Wang and J. Fang. 2020. Genome-wide iden- tification and characterization of gibberellin metabolic and signal transduction (GA MST) pathway mediating seed and berry development (SBD) in grape (Vitis vinifera L.). BMC Plant Biol. 20: 384. DOI: 10.1186/s12870-020-02591-1.

Wang, X., W. Wang, J. Wang, N. Zhang, L. Yang, L. Cai, S. Hussain, Y. Chang and S. Wang. 2019. Class III OFPs function in the ER signaling pathway to regulate plant growth and development in Arabidopsis. J. Plant Interact. 14: 4553.

Yamada, M. and A. Sato. 2016. Advances in table grape breeding in Japan. Breed. Sci. 66: 34-45.

Yamada, M., H. Yamane, A. Sato, H. Iwanami, K. Yoshinaga, T. Ozawa, N. Mitani, M. Shiraishi, M. Yoshioka, I. Nakajima, M. Nakano and R. Nakaune. 2008. New grape cultivar 'Shine Muscat'. Bull. Nat. Inst. Fruit Tree Sci. 7: 21-38.

Yamada, M., H. Yamane, K. Yoshinaga, N. Hirakawa, A. Kurihara, H. Iwanami, K. Nagata, A. Sato, T. Ozawa, T. Sumi, T. Hirabayashi, M. Kakutani and I. Nakajima. 2003. New grape cultivar 'Honey Venus'. Bull. Nat. Inst. Fruit Tree Sci. 2: 53-63.

Yamaguchi, S. 2008. Gibberellin metabolism and its regulation. Annu. Rev. Plant Biol. 59: 225-251.

Yoshimura, D., A. Sato, D. Ito, H. Nakamoto, A. Okamoto, M. Miyazaki, K. Kita, J. Ueda and K. Yonemori. 2021. Seedlessness induction by gibberellic acid in large-sized berry grapes. Hort. Res. (Japan) 20: 199-205 (In Japanese with English abstract). 